\title{
Van der Waals coefficients beyond the classical shell model
}

\author{
Jianmin Tao, ${ }^{1, a)}$ Yuan Fang, ${ }^{2}$ Pan Hao, ${ }^{2}$ G. E. Scuseria,${ }^{3}$ Adrienn Ruzsinszky, ${ }^{4}$ \\ and John P. Perdew 4 \\ ${ }^{1}$ Department of Chemistry, University of Pennsylvania, Philadelphia, Pennsylvania 19104-6323, USA \\ ${ }^{2}$ Department of Physics and Engineering Physics, Tulane University, New Orleans, Louisiana 70118, USA \\ ${ }^{3}$ Department of Chemistry and Department of Physics and Astronomy, Rice University, Houston, \\ Texas 77251-1892, USA and Department of Chemistry, Faculty of Science, King Abdulaziz University, \\ Jeddah 21589, Saudi Arabia \\ ${ }^{4}$ Department of Physics, Temple University, Philadelphia, Pennsylvania 19122, USA
}

(Received 10 October 2014; accepted 11 December 2014; published online 12 January 2015)

\begin{abstract}
Van der Waals (vdW) coefficients can be accurately generated and understood by modelling the dynamic multipole polarizability of each interacting object. Accurate static polarizabilities are the key to accurate dynamic polarizabilities and vdW coefficients. In this work, we present and study in detail a hollow-sphere model for the dynamic multipole polarizability proposed recently by two of the present authors (JT and JPP) to simulate the vdW coefficients for inhomogeneous systems that allow for a cavity. The inputs to this model are the accurate static multipole polarizabilities and the electron density. A simplification of the full hollow-sphere model, the single-frequency approximation (SFA), circumvents the need for a detailed electron density and for a double numerical integration over space. We find that the hollow-sphere model in SFA is not only accurate for nanoclusters and cage molecules (e.g., fullerenes) but also yields vdW coefficients among atoms, fullerenes, and small clusters in good agreement with expensive time-dependent density functional calculations. However, the classical shell model (CSM), which inputs the static dipole polarizabilities and estimates the static higher-order multipole polarizabilities therefrom, is accurate for the higher-order vdW coefficients only when the interacting objects are large. For the lowest-order vdW coefficient $C_{6}$, SFA and CSM are exactly the same. The higher-order $\left(C_{8}\right.$ and $\left.C_{10}\right)$ terms of the vdW expansion can be almost as important as the $C_{6}$ term in molecular crystals. Application to a variety of clusters shows that there is strong non-additivity of the long-range vdW interactions between nanoclusters. (C) 2015 AIP Publishing LLC. [http://dx.doi.org/10.1063/1.4905259]
\end{abstract}

\section{INTRODUCTION}

Van der Waals (vdW) attraction ${ }^{1}$ is an important longrange nonlocal correlation, arising from instantaneous charge fluctuations on each density piece that may or may not belong to the same system. It affects the properties of materials and many physical and chemical processes. Typical examples include lattice constants, ${ }^{2,3}$ cohesive energies, ${ }^{2,4}$ sublimation energies, ${ }^{5,6}$ melting and boiling points, disorder effect of polymers and higher-order geometry configurations of DNA, ${ }^{7}$ and physical adsorption of atoms and molecules on surfaces. ${ }^{8-10}$ A better understanding and accurate prediction of the vdW interaction will benefit many branches of science (e.g., chemistry, physics, materials science, and biology) and technology (e.g., molecular drug design, hydrogen storage, ${ }^{11}$ purification, catalyst activity improvement).

The vdW energy between two well-separated spherical densities can be written as ${ }^{12}$

$$
E_{\mathrm{vdW}}=-\frac{C_{6}}{d^{6}}-\frac{C_{8}}{d^{8}}-\frac{C_{10}}{d^{10}}-\cdots,
$$

where $d$ is the distance between the centers of the two densities. According to second-order perturbation theory, the $\mathrm{vdW}$ coefficients $C_{2 k}$ may be expressed in terms of the dynamic

\footnotetext{
a)Electronic address: jianmint@sas.upenn.edu. URL: http://www.sas.upenn. edu/ jianmint/
}

multipole polarizability ${ }^{13} \alpha_{l}(i u)$, i.e.,

$$
C_{2 k}^{\mathrm{AB}}=\frac{(2 k-2) !}{2 \pi} \sum_{l_{1}=1}^{k-2} \frac{1}{\left(2 l_{1}\right) !\left(2 l_{2}\right) !} \int_{0}^{\infty} d u \alpha_{l_{1}}^{\mathrm{A}}(i u) \alpha_{l_{2}}^{\mathrm{B}}(i u) .
$$

Here, $i u$ is imaginary frequency, $l_{2}=k-l_{1}-1$, with $l=1$ (dipole), $l=2$ (quadrupole), $l=3$ (octupole), etc.

The dynamic multipole polarizability is defined as the linear response of a system to a weak, time-dependent external electric field ${ }^{14}$ oscillating at frequency $\omega$. It can be evaluated with the sum-over-states perturbation expression ${ }^{15}$

$$
\alpha_{l}(i u)=\sum_{n=1}^{\infty} \frac{f_{n}^{l}}{\left(E_{n}-E_{0}\right)^{2}+u^{2}},
$$

with $E_{n}-E_{0}$ being the transition energy from the ground state $\Psi_{0}$ to the excited state $\Psi_{n}$ and $f_{n}^{l}$ being the oscillator strength. This approach has been widely used in wavefunctionbased MBPT (many-body perturbation theory), ${ }^{16}$ coupled cluster, ${ }^{17} \mathrm{CI}$ (configuration interaction) ${ }^{18}$ methods, or their combinations. ${ }^{19,20}$ While these methods are highly accurate, they are computationally demanding. The dynamic multipole polarizability can be alternatively expressed in terms of the density response function ${ }^{21,22}$ defined by

$$
\chi_{n n}\left(\mathbf{r}, \mathbf{r}^{\prime} ; i u\right)=i \int_{0}^{\infty} d \tau e^{-u \tau}\left\langle\Psi\left|\left[\hat{n}(\mathbf{r}, \tau), \hat{n}\left(\mathbf{r}^{\prime}\right)\right]\right| \Psi\right\rangle .
$$


In general, the response function $\chi_{n n}\left(\mathbf{r}, \mathbf{r}^{\prime} ; i u\right)$ is a highly nonlocal function in both space and time. It can be evaluated from the wave function-based methods ${ }^{23}$ or calculated efficiently from less accurate but improvable time-dependent density functional theory (TDDFT). ${ }^{24}$

However, application of these approaches to nanosize or larger systems presents a computational challenge, due to two facts. First, distorted or perturbed states are very sensitive to the basis set. To guarantee computational reliability, a large basis set must be used. ${ }^{25}$ Second, wave function-based or first-principles calculations of the vdW coefficients require knowledge of the dynamic multipole polarizability at each imaginary frequency point. To overcome this computational difficulty, many models ${ }^{2,26-29}$ for the dynamic polarizability have been proposed. But most of them have been devoted to the dipole polarizability.

Recently, it has been shown ${ }^{2,29,30}$ that the higher-order contribution is much more important than previously believed ${ }^{4}$ ( $20 \%$ of the leading-order term $C_{6} / d^{6}$ ). For lattice constants and cohesive energies of alkali metals, we found ${ }^{2}$ that the higher-order terms such as $C_{8}$ and $C_{10}$ can be as large as $50 \%$ of the leading-order contribution. For molecular crystals, we find in Sec. VI that the $C_{8}$ and $C_{10}$ terms can be as important as the $C_{6}$ term. Although higher-order contributions are important in the simulation of the $\mathrm{vdW}$ interaction, it is very difficult to estimate higher-order coefficients, without empirical fitting. Consequently, they are ignored in most vdW calculations. We proposed a simple model ${ }^{2,29,31}$ for the dynamic dipole polarizability, from which $C_{6}$ can be obtained accurately. This model was then extended to the multipole polarizability. The extension is successful, because higher-order vdW coefficients such as $C_{8}$ and $C_{10}$ generated with the extended model can achieve the same accuracy (3\% mean absolute relative error for free atom pairs) as the original model for the dipole polarizability. For convenience, we combine the simple model and its extension and call the combination the solid-sphere model. A limitation of the solid-sphere model is that it is only valid for a density that has no cavity. For any density with a cavity, the model is unsuitable. To overcome this limitation, Tao and Perdew have developed ${ }^{30}$ a hollow-sphere model to describe the dynamic multipole polarizability for a shell of density. This model can be further simplified with the singlefrequency approximation (SFA), in which the vdW coefficients can be expressed without involving numerical integrations in space and imaginary frequency. When the cavity vanishes, the hollow-sphere model reduces to the solid-sphere model. The versatility of the hollow-sphere model allows us, on the same footing, to study pair interactions between systems that may or may not have a cavity and that may be small or truly large. Application of the hollow-sphere model in the singlefrequency approximation to sodium clusters and fullerenes shows that the single-frequency approximation yields the $\mathrm{vdW}$ coefficients in good agreement (about 7\%) with expensive time-dependent Hartree-Fock (TDHF) theory and TDDFT.

The starting point of the hollow-sphere model is the classical conducting spherical-shell model, ${ }^{32-34}$ which was derived from the classical conducting metallic shell of density uniform inside and zero outside the shell. This classical shell model (CSM) has been used to study the asymptotic size- dependence of the vdW coefficients for fullerenes ${ }^{30,33}$ and clusters with no cavity ${ }^{30}$ and to investigate the convergence of the vdW asymptotic series of Eq. (1). ${ }^{34}$ In this work, we will make a detailed comparison of these models and present the vdW coefficients for alkali clusters and fullerenes.

In Ref. 30, we studied $C_{6}$ and its non-additivity for several pairs of clusters: $\mathrm{Na}_{\mathcal{N}}-\mathrm{Na}_{\mathcal{N}}$, fullerene-fullerene, $\mathrm{Si}_{\mathcal{N}}-\mathrm{Si}_{\mathcal{N}}$, $\mathrm{Ge}_{\mathcal{N}}-\mathrm{Ge}_{\mathcal{N}}, \mathrm{Na}_{\mathcal{N}}$-fullerene, and hydrogen-terminated silicon clusters $\mathrm{Si}_{\mathcal{N}}-\mathrm{H}_{\mathcal{M}}$. Here, we will explain our formalism in detail and apply it further to Li-fullerene, K-fullerene, $\mathrm{Li}_{\mathcal{N}}-\mathrm{Li}_{\mathcal{N}}$, $\mathrm{K}_{\mathcal{N}}-\mathrm{K}_{\mathcal{N}}, \mathrm{Na}_{\mathcal{N}}-\mathrm{K}_{\mathcal{N}}$, and $\mathrm{Al}_{\mathcal{N}}-\mathrm{Al}_{\mathcal{N}}$ cluster pairs. For large clusters, ours may be the only accurate method that is practical. Therefore, it can serve as a benchmark for other practical methods.

It is well-known that semilocal and hybrid density functionals, employed in a ground-state calculation, cannot capture the long-range $\mathrm{vdW}$ interaction (although some $\mathrm{e}^{35,36}$ capture the intermediate range of this interaction). Long-range $\mathrm{vdW}$ corrections to semilocal functionals are now widely used, and the most popular ones capture some non-additive effects, although they are not expected to be reliable for metals. These long-range corrections include fully nonlocal correlation energy functionals (see Refs. 37 and 28), pairwise interactions between effective atoms-in-molecules, ${ }^{38,40}$ and a model of interacting harmonic oscillators. ${ }^{39} \mathrm{It}$ is also possible to sum up the whole vdW series approximately, although the result is still only an asymptotic expression valid for large $d{ }^{32}$

\section{CLASSICAL SHELL MODEL}

Consider a classical conducting spherical shell of uniform density with outer radius $R$ and shell thickness $t$. The exact dynamic multipole polarizability of the shell is given by ${ }^{41}$

$$
\begin{aligned}
\alpha_{l}^{\mathrm{csm}}(i u) & =\left[R^{2 l+1} \frac{\epsilon-1}{\epsilon+(l+1) / l}\right] \frac{1-\rho_{l}}{1-\beta_{l} \rho_{l}}, \\
\beta_{l} & =\frac{(\epsilon-1)^{2}}{[\epsilon+l /(l+1)][\epsilon+(l+1) / l]}, \\
\rho_{l} & =\left(\frac{R-t}{R}\right)^{2 l+1},
\end{aligned}
$$

where $\epsilon$ is the dielectric function of the isotropic medium and $\rho_{l}$ defines the "shape" of the shell. The quantity in brackets of Eq. (5) is the dynamic multipole polarizability of a classical conducting metallic sphere of uniform density, while the remaining part arises from the coupling of the sphere and cavity plasmon vibrations, as seen below. Like the classical conducting metallic sphere, the classical conducting metallic spherical shell has a sharp physical boundary for the density.

In the long wavelength or small wave-vector limit, the dielectric function of the isotropic medium is

$$
\epsilon(i u)=1+\omega_{p}^{2} / u^{2},
$$

where $\omega_{p}=\sqrt{4 \pi n}$ is the plasmon frequency of the extended uniform electron gas, with $n$ being the electron density. (Hartree atomic units are used.) Substituting Eq. (8) into Eqs. 
(5) and (6) leads to a simple expression

$$
\begin{aligned}
\alpha_{l}^{\mathrm{csm}}(i u) & =\left(R^{2 l+1} \frac{\omega_{l}^{2}}{\omega_{l}^{2}+u^{2}}\right) \frac{1-\rho_{l}}{1-\beta_{l} \rho_{l}}, \\
\beta_{l} & =\frac{\omega_{l}^{2} \tilde{\omega}_{l}^{2}}{\left(\omega_{l}^{2}+u^{2}\right)\left(\tilde{\omega}_{l}^{2}+u^{2}\right)} .
\end{aligned}
$$

Here, $\omega_{l}=\omega_{p} \sqrt{l /(2 l+1)}$ is the mode plasmon vibrational frequency of the sphere, and $\tilde{\omega}_{l}=\omega_{p} \sqrt{(l+1) /(2 l+1)}$ is the mode plasmon vibrational frequency of the cavity. When the cavity vanishes (i.e., $t=R$ ), the dynamic multipole polarizability of the classical shell reduces to that of a classical solid sphere.

From Eq. (10), we see that, in the zero-frequency limit, $\beta_{l}=1$. Thus we have

$$
\alpha_{l}^{\mathrm{csm}}(0)=R^{2 l+1},
$$

which is equivalent to

$$
\alpha_{1}^{\mathrm{csm}}(0)=\left[\alpha_{2}^{\mathrm{csm}}(0)\right]^{3 / 5}=\left[\alpha_{3}^{\mathrm{csm}}(0)\right]^{3 / 7}=\cdots .
$$

Thus, in the classical model, higher-order static multipole polarizabilities $\alpha_{l}^{\mathrm{csm}}(0)$ are calculated from $\alpha_{1}^{\mathrm{csm}}(0)$, which is taken to be the accurate static dipole polarizability evaluated from wave function-based ab initio or first-principles methods with single-point calculation. The shell thickness affects the frequency dependence of the dynamic multipole polarizability via $\rho_{l}$ and $\beta_{l}$. In the high-frequency (i.e., $\tilde{\omega}_{l} / u \ll 0$ ) limit, $\beta_{l}=0$ and $\alpha_{l}^{\text {csm }}(i u)=R^{2 l+1} \omega_{l}^{2}\left(1-\rho_{l}\right) / u^{2}$, which can be recast into the form

$$
\alpha_{l}(i u)=\frac{l\left\langle r^{2 l-2}\right\rangle}{u^{2}},
$$

the exact high-frequency limit. ${ }^{27}$ For inhomogeneous densities, $\alpha_{l}^{\operatorname{csm}}(i u)=R^{2 l+1} \omega_{l}^{2}\left(1-\rho_{l}\right) / u^{2}$ only satisfies Eq. (13) approximately. Here, $\left\langle r^{2 l-2}\right\rangle=\int_{0}^{\infty} d r 4 \pi r^{2 l} n$ is the expectation value. For the classical shell model, the density $n$ in $R-t<r<$ $R$ is defined by

$$
n=N / V, \quad V=(4 \pi / 3) R^{3}-(4 \pi / 3)(R-t)^{3},
$$

with $N$ being the number of valence electrons and $V$ being the volume of the shell.

The vdW coefficients within the classical model can be obtained by substituting Eq. (9) into Eq. (2). The result is

$$
C_{2 k}=\frac{(2 k-2) !}{2 \pi} \sum_{l_{1}=1}^{k-2} \frac{\left(R_{\mathrm{A}}\right)^{2 l_{1}+1}\left(R_{\mathrm{B}}\right)^{2 l_{2}+1}}{\left(2 l_{1}\right) !\left(2 l_{2}\right) !} H_{l_{1}, l_{2}}^{\mathrm{AB}} I_{l_{1}, l_{2}}^{\mathrm{AB}},
$$

where

$$
\begin{aligned}
& H=\left(\omega_{l_{1}}^{\mathrm{A}}\right)^{2}\left(\omega_{l_{2}}^{\mathrm{B}}\right)^{2}\left(1-\rho_{l_{1}}^{\mathrm{A}}\right)\left(1-\rho_{l_{2}}^{\mathrm{B}}\right), \\
& I=\frac{\pi}{2} \frac{\left[Q+S+f\left(a_{l_{1}}^{\mathrm{A}}, b_{l_{1}}^{\mathrm{A}} ; a_{l_{2}}^{\mathrm{B}}, b_{l_{2}}^{\mathrm{B}}\right)+f\left(a_{l_{2}}^{\mathrm{B}}, b_{l_{2}}^{\mathrm{B}} ; a_{l_{1}}^{\mathrm{A}}, b_{l_{1}}^{\mathrm{A}}\right)\right]}{P\left(a_{l_{1}}^{\mathrm{A}}+b_{l_{1}}^{\mathrm{A}}\right)\left(a_{l_{1}}^{\mathrm{A}}+b_{l_{2}}^{\mathrm{B}}\right)\left(a_{l_{2}}^{\mathrm{B}}+b_{l_{1}}^{\mathrm{A}}\right)\left(a_{l_{2}}^{\mathrm{B}}+b_{l_{2}}^{\mathrm{B}}\right)}, \\
& Q=\left(a_{l_{1}}^{\mathrm{A}} b_{l_{1}}^{\mathrm{A}}\right)^{2} D\left(a_{l_{2}}^{\mathrm{B}}, b_{l_{2}}^{\mathrm{B}}\right)+\left(a_{l_{2}}^{\mathrm{B}} b_{l_{2}}^{\mathrm{B}}\right)^{2} D\left(a_{l_{1}}^{\mathrm{A}}, b_{l_{1}}^{\mathrm{A}}\right), \\
& S=\left[\left(\tilde{\omega}_{l_{1}}^{\mathrm{A}}\right)^{2}+\left(\tilde{\omega}_{l_{2}}^{\mathrm{B}}\right)^{2}\right] a_{l_{1}}^{\mathrm{A}} a_{l_{2}}^{\mathrm{B}} b_{l_{1}}^{\mathrm{A}} b_{l_{2}}^{\mathrm{B}}\left(a_{l_{1}}^{\mathrm{A}}+a_{l_{2}}^{\mathrm{B}}+b_{l_{1}}^{\mathrm{A}}+b_{l_{2}}^{\mathrm{B}}\right), \\
& P=D\left(a_{l_{1}}^{\mathrm{A}}, a_{l_{2}}^{\mathrm{B}}\right) D\left(b_{l_{1}}^{\mathrm{A}}, b_{l_{2}}^{\mathrm{B}}\right),
\end{aligned}
$$

$$
\begin{aligned}
& a_{l}=\frac{1}{\sqrt{2}}\left[\left(\omega_{l}^{2}+\tilde{\omega}_{l}^{2}\right)-\sqrt{\left(\omega_{l}^{2}-\tilde{\omega}_{l}^{2}\right)^{2}+4 \rho_{l} \omega_{l}^{2} \tilde{\omega}_{l}^{2}}\right]^{1 / 2}, \\
& b_{l}=\frac{1}{\sqrt{2}}\left[\left(\omega_{l}^{2}+\tilde{\omega}_{l}^{2}\right)+\sqrt{\left(\omega_{l}^{2}-\tilde{\omega}_{l}^{2}\right)^{2}+4 \rho_{l} \omega_{l}^{2} \tilde{\omega}_{l}^{2}}\right]^{1 / 2},
\end{aligned}
$$

with $D$ and $f$ being defined by $D(x, y)=x y(x+y)$ and $f(p, q ; s, t)=\left(\tilde{\omega}_{l_{1}}^{\mathrm{A}}\right)^{2}\left(\tilde{\omega}_{l_{2}}^{\mathrm{B}}\right)^{2}(p+q)\left(p q+2 s t+s^{2}+t^{2}\right)$. In these expressions, the superscripts (A, B) and subscripts $\left(l_{1}, l_{2}\right)$ have been dropped without ambiguity.

\section{HOLLOW-SPHERE MODEL}

As discussed above, the classical shell model is suitable for nanoscale or larger systems, in which the electron density is slowly varying. For smaller systems, it is not valid anymore, because the density in small systems is not slowly varying. In this case, an obvious problem with the classical shell model is that classical relationship (12) for the static multipole polarizability, which is rooted in the fact that the classical shell has a sharp physical boundary for all orders, can be seriously wrong. As a result, the classical model may yield a reasonable estimate for $C_{6}$, but the error for $C_{8}$ and $C_{10}$ can be large. This expectation has been confirmed in the extreme case of free atom pairs, for which it is found here (Table I for summarized results and Tables S1-S3 for details ${ }^{42}$ ) that the mean absolute relative error is only $9 \%$ for $C_{6}$, but the errors for $C_{8}$ and $C_{10}$ are unacceptably large (50\% for $C_{8}$ and $66 \%$ for $C_{10}$ ). Furthermore, application to a $\mathrm{C}_{60}-\mathrm{C}_{60}$ pair shows ${ }^{30}$ that while the classical shell model yields $C_{6}$ and $C_{8}$ in good agreement with the TDHF values, there is a significant deviation for $C_{10}$. Therefore, the classical shell model must be generalized.

\section{A. Full hollow-sphere model}

A method beyond the classical shell model has been proposed $^{30}$ to describe the dynamic multipole polarizability of a density that allows for a cavity. Since the density may be diffuse from a surface formed by the nuclear framework, it has no sharp physical boundary. For such a density, as sketched in Fig. 1, the dynamic multipole polarizability was assumed to be

$$
\alpha_{l}^{\mathrm{hsm}}(i u)=\frac{2 l+1}{4 \pi d_{l}} \int_{R_{l^{-}-t_{l}}}^{R_{l}} d^{3} r\left(\frac{r^{2 l-2} d_{l}^{4} \omega_{l}^{2}}{d_{l}^{4} \omega_{l}^{2}+u^{2}}\right) \frac{1}{1-\tilde{\beta}_{l} \tilde{\rho}_{l}},
$$

TABLE I. Mean absolute relative error of the vdW coefficients with respect to highly accurate wave function-based methods for 78 atom-atom pair interactions calculated from the full hollow-sphere or solid-sphere model of Eq. (33), SFA of Eq. (37), and the classical shell model of Eq. (15), respectively. For atoms, we set $t_{l}=R_{l}$. Detailed comparison and accurate reference values can be found from the supplementary material. ${ }^{42}$ Note the striking failure of the CSM for $C_{8}$ and $C_{10}$ between small objects.

\begin{tabular}{lcrr}
\hline \hline & $C_{6}$ & $C_{8}$ & $C_{10}$ \\
\hline Hollow-sphere model (\%) & $3^{\text {a }}$ & $3^{\text {a }}$ & $3^{\text {a }}$ \\
SFA (\%) & 9 & 7 & 15 \\
Classical shell model (\%) & 9 & 50 & 66 \\
\hline
\end{tabular}

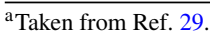




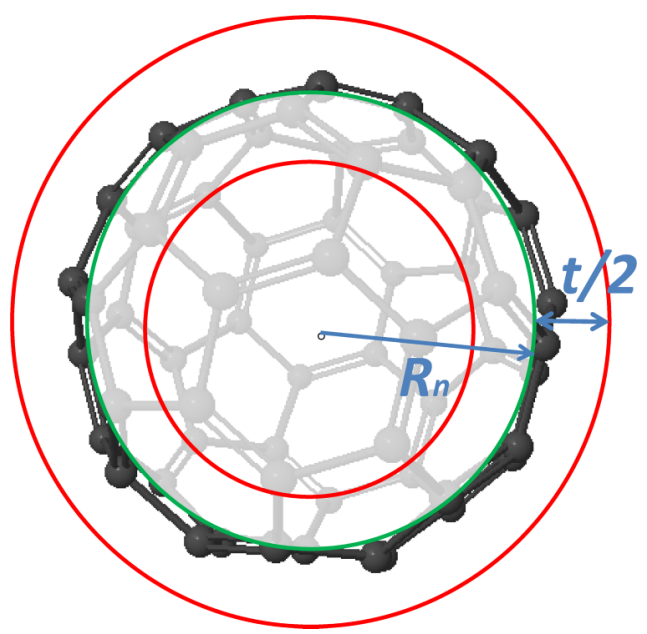

FIG. 1. Shell structure of a fullerene cluster. $R_{n}$ is the average radius of the nuclear framework, and $t$ is the shell thickness.

$$
\begin{aligned}
& \tilde{\beta}_{l}=\frac{d_{l}^{8} \omega_{l}^{2} \tilde{\omega}_{l}^{2}}{\left(d_{l}^{4} \omega_{l}^{2}+u^{2}\right)\left(d_{l}^{4} \tilde{\omega}_{l}^{2}+u^{2}\right)}, \\
& \tilde{\rho}_{l}=\left(\frac{R_{l}-t_{l}}{R_{l}}\right)^{2 l+1},
\end{aligned}
$$

where $R_{l}$ is the effective (or vdW) outer radius of the shell, while $d_{l}$ is a parameter introduced to ensure that the correct static and high-frequency limit conditions are satisfied. Here, the last factor in Eq. (23) adds the cavity effect to the solid sphere model (see Ref. 29 or Eq. (2) of Ref. 30). It essentially stems from the last fraction of Eq. (5), but with the constant electron density in $\beta_{l}$ replaced by the local density. Similar to the classical shell model, ${ }^{33,43}$ the shell thickness $t_{l}$ is defined for a single-walled fullerene by

$$
R_{l}=R_{n}+t_{l} / 2 .
$$

(Note that in the classical shell model, both $R$ and $t$ are $l$-independent.) $R_{n}$ is the average radius of the nuclear framework. Given $R_{l}$ and the geometric input $R_{n}$, we can find the geometric descriptor $t_{l} \leq R_{l}$ that distinguishes a hollow sphere from a solid sphere. Clearly, the $l$-dependent vdW shell coincides with the classical conducting shell $\left(R_{l}=R\right.$ and $\left.t_{l}=t\right)$ only when the density is uniform within the shell and zero outside the shell (so that the parameter $d_{l}=1$ ) and when the higher-order static polarizability satisfies Eq. (12). The two parameters $R_{l}$ and $d_{l}$ are determined following Ref. 29. In the zero-frequency $(u \rightarrow 0)$ limit, $\alpha_{l}^{\mathrm{hsm}}(i u)$ of Eq. (23) reduces to

$$
\alpha_{l}^{\mathrm{hsm}}(i u)=R_{l}^{2 l+1} / d_{l},
$$

while in the high-frequency $\left(u / \omega_{l}, u / \tilde{\omega}_{l} \gg 1\right)$ limit, $\tilde{\beta}_{l} \rightarrow 0$ and

$$
\alpha_{l}^{\mathrm{hsm}}(i u)=l d_{l}^{3} \int_{R_{l}-t_{l}}^{R_{l}} d^{3} r r^{2 l-2} n(\mathbf{r}) / u^{2} .
$$

This means that to restore the exact high-frequency limit, the excess contribution of the local plasmon frequency outside the shell of radius $R_{l}$ must be cutoff. To recover the correct static and high-frequency [Eq. (13)] limits, $R_{l}$ and $d_{l}$ must satisfy the following two equations:

$$
R_{l}=\left[d_{l} \alpha_{l}^{\mathrm{hsm}}(0)\right]^{1 /(2 l+1)},
$$

$$
d_{l}=\left\{\int_{0}^{\infty} d r 4 \pi r^{2 l} n(r) / \int_{R_{l^{-}-t_{l}}}^{R_{l}} d r 4 \pi r^{2 l} n(r)\right\}^{1 / 3} .
$$

Since the two equations are coupled together, they must be solved self-consistently. $\alpha_{l}^{\mathrm{hsm}}(0)$ is the accurate static multipole polarizability $\alpha_{l}(0)$ that can be calculated accurately from $a b$ initio or first-principles methods, which have been documented in the literature. ${ }^{1,44}$ Equations (23)-(30) constitute the full hollow-sphere model. From Eq. (23) we see that when the cavity vanishes (i.e., $t_{l}=R_{l}, \rho_{l}=0$ ), the hollow-sphere model reduces to the solid-sphere model. It was shown ${ }^{29}$ that the solid-sphere is not only exact in the uniform-gas limit but also accurate for the $\mathrm{H}$ and $\mathrm{He}$ atoms. The hollow-sphere model may be regarded as an interpolation for imaginary frequency between exact zero- and high-frequency limits. It may be also regarded as an interpolation for the density between the uniform-gas limit (paradigm in condensed matter physics) and one- or two-electron densities (paradigm in quantum chemistry).

Local polarizability models such as those of Refs. 2, 28, and 29 can be unsuitable ${ }^{33}$ to systems that have a cavity. To see this, we just set $\tilde{\rho}_{l}$ to zero in Eq. (23). This leads (at least for $l=1$ ) to a nearly local polarizability model for the dynamic polarizability

$$
\alpha_{l}^{\mathrm{lp}}(i u)=\frac{2 l+1}{4 \pi d_{l}} \int_{R_{l^{-} t_{l}}}^{R_{l}} d^{3} r\left(\frac{r^{2 l-2} d_{l}^{4} \omega_{l}^{2}}{d_{l}^{4} \omega_{l}^{2}+u^{2}}\right) .
$$

The spatial integration limit is from $R_{l}-t_{l}$ to $R_{l}$, because only the density within the shell contributes, while the contribution from the density outside the shell has been cutoff. In the static $(u \rightarrow 0)$ limit, we have

$$
\alpha_{l}^{\mathrm{lp}}(0)=\left[R_{l}^{2 l+1}-\left(R_{l}-t_{l}\right)^{2 l+1}\right] / d_{l} .
$$

For a fullerene $\mathrm{C}_{60}$ molecule, the density is quite uniform due to the electron delocalization, to a good approximation, so we assume $d_{l}=1$. For such a molecule, $R_{1}=8.11$ bohrs, $t_{l}=2.77$ bohrs. Thus the static dipole polarizability in the local polarizability model is $\alpha_{1}^{\mathrm{lp}}(0)=381 \mathrm{bohrs}^{3}$, much smaller than the TDDFT value 534 bohrs $^{3}$. (The experimental value is $516 \pm 54$ bohrs $^{3}$.)

The vdW coefficients from the full hollow-sphere model may be expressed as ${ }^{30}$

$$
\begin{aligned}
C_{2 k}= & \frac{(2 k-2) !}{32 \pi^{3}} \sum_{l_{1}=1}^{k-2} \frac{\left(2 l_{1}+1\right)\left(2 l_{2}+1\right)}{\left(2 l_{1}\right) !\left(2 l_{2}\right) !}\left(d_{l_{1}}^{\mathrm{A}} d_{l_{2}}^{\mathrm{B}}\right)^{3} \\
& \times \int_{R_{l_{1}}^{\mathrm{A}}-t_{l_{1}}^{\mathrm{A}}}^{R_{l_{1}}^{\mathrm{A}}} d^{3} r_{\mathrm{A}} r_{\mathrm{A}}^{2 l_{1}-2}\left(\omega_{l_{1}}^{\mathrm{A}}\right)^{2} \\
& \times \int_{R_{l_{2}}^{\mathrm{B}}-t_{l_{2}}^{\mathrm{B}}}^{R_{l_{2}}^{\mathrm{B}}} d^{3} r_{\mathrm{B}} r_{\mathrm{B}}^{2 l_{2}-2}\left(\omega_{l_{2}}^{\mathrm{B}}\right)^{2} I_{l_{1} l_{2}}^{\mathrm{AB}},
\end{aligned}
$$

where $I_{l_{1} l_{2}}^{\mathrm{AB}}$ is defined by Eqs. (17)-(22) but with $\omega_{l}$ and $\tilde{\omega}_{l}$ replaced by $d_{l}^{2} \omega_{l}$ and $d_{l}^{2} \tilde{\omega}_{l}, \rho_{l}$ of Eq. (7) by $\tilde{\rho}_{l}$ of Eq. (25), and the average valence electron density by the true electron density. This expression is quite versatile. It allows us to treat different situations. 
In this work, we will present results mainly for the hollow-sphere model in the single-frequency approximation, as defined in Sec. III B.

\section{B. Single-frequency approximation}

The full hollow-sphere model requires knowledge of the true electron density. This requirement can be avoided with the SFA, in which we assume that (i) only valence electrons are polarizable, and (ii) for each $l$, the (vdW or effective) electron density is uniform (i.e., single plasmon vibrational frequency) within the shell and zero outside the shell. The SFA can be regarded either as a simplification of the full hollow-sphere model or as an extension of the classical shell model. With assumption (i), we can replace the all-electron density by the valence electron density in Eq. (30), and with assumption (ii), we replace the local valence electron density by the average valence electron density, leading to $d_{l}=1$. The dynamic multipole polarizability in SFA takes the form

$$
\alpha_{l}^{\mathrm{SFA}}(i u)=\left(R_{l}^{2 l+1} \frac{\omega_{l}^{2}}{\omega_{l}^{2}+u^{2}}\right) \frac{1-\tilde{\rho}_{l}}{1-\tilde{\beta}_{l} \tilde{\rho}_{l}},
$$

with $R_{l}$ given by

$$
R_{l}=\left[\alpha_{l}^{\mathrm{SFA}}(0)\right]^{1 / 2 l+1},
$$

and $\tilde{\beta}_{l}$ and $\tilde{\rho}_{l}$ defined by Eqs. (24) and (25), but with the local electron density replaced by the average valence electron density and $d_{l}=1$. Since both the full hollow-sphere model and SFA recover the correct static limit, $\alpha_{l}^{\mathrm{SFA}}(0)=\alpha_{l}^{\mathrm{hsm}}(0)=\alpha_{l}(0)$. For clarity, the superscript in $\alpha_{l_{1}}^{\mathrm{SFA}}(0)$ will be dropped without ambiguity. Although the functional form of the dynamic multipole polarizability in SFA is the same as that of the classical shell model [Eq. (9)], there is an important distinction between these two methods. In the classical shell model, we only need the accurate static dipole polarizability as input, while the higher-order multipole polarizabilities will be estimated from the classical relationship (12). This leads to $R_{l}=R$ and $t_{l}=t$ for any order $l$. In SFA, the inputs are the accurate static dipole as well as higher-order multipole polarizabilities. Therefore, in SFA, the vdW radius $R_{l}$ and the effective thickness $t_{l}$ of the shell have a weak $l$-dependence. Because we are making a simplified model that satisfies exact constraints and limits, $R_{l}$ and $t_{l}$ have only approximate physical meanings. The average valence electron density is calculated from

$$
n_{l}=N / V_{l}, \quad V_{l}=(4 \pi / 3) R_{l}^{3}-(4 \pi / 3)\left(R_{l}-t_{l}\right)^{3},
$$

which is also $l$-dependent. Thus, the average valence electron density and the high-frequency limit in SFA are different from those in the classical shell model.

In SFA, the expression for the vdW coefficients can be simplified as

$$
C_{2 k}=\frac{(2 k-2) !}{2 \pi} \sum_{l_{1}=1}^{k-2} \frac{\alpha_{l_{1}}^{\mathrm{A}}(0) \alpha_{l_{2}}^{\mathrm{B}}(0)}{\left(2 l_{1}\right) !\left(2 l_{2}\right) !} H_{l_{1}, l_{2}}^{\mathrm{AB}} I_{l_{1}, l_{2}}^{\mathrm{AB}} .
$$

Here, $H_{l_{1}, l_{2}}^{\mathrm{AB}}$ and $I_{l_{1}, l_{2}}^{\mathrm{AB}}$ have the same expression as those in the classical shell model [Eqs. (16)-(22)], but the shape function and average valence electron density of Eqs. (7) and (14) are replaced by Eqs. (25) and (36), respectively. Eq. (37) yields the same $C_{6}$ coefficients as the CSM does.

\section{IV. "SHAPE" EFFECT UPON THE DYNAMIC MULTIPOLE POLARIZABILITY}

From Eq. (34), we see that the outer radius and the shell thickness are two important factors that affect the dynamic multipole polarizability independently. There are many studies on the cluster size dependence of the static polarizability. This size dependence has a direct effect on the dynamic multipole polarizability, because a crucial input in the present theory is the static multipole polarizability. In order to have a better understanding of the vdW interaction, we investigate another effect, the geometry or "shape" effect.

(i) $N$ and $R_{l}$ are fixed, but $t_{l}$ varies up to $R_{l}$. For a given system, the number of valence electrons $N$ is fixed. The average valence electron density is then determined by the outer radius $R_{l}$ and the shell thickness $t_{l}$. Let us first consider a case in which $N$ and $R_{l}$ are fixed. When $t_{l} \rightarrow R_{l}$, hollow-sphere model (23) reduces to the solidsphere model

$$
\alpha_{l}^{\mathrm{ssm}}(i u)=\frac{2 l+1}{4 \pi d_{l}} \int_{0}^{R_{l}} d^{3} r \frac{r^{2 l-2} d_{l}^{4} \omega_{l}^{2}}{d_{l}^{4} \omega_{l}^{2}+u^{2}},
$$

with $d_{l}$ being given by Eq. (30). Clearly, the density of a solid sphere is lower than that of a spherical shell. In SFA, the solid-sphere model takes a very simple form [Eq. (34) with $\left.\tilde{\rho}_{l}=0\right]$. The average electron density, which enters the formula via $\omega_{l}$, is calculated from

$$
n=N / V_{l}, \quad V_{l}=\frac{4 \pi}{3} R_{l}^{3} .
$$

Figure 2 shows the shell thickness dependence of the dynamic multipole polarizability at imaginary frequency points $u=0.1,0.5,2.0$, obtained from the CSM [Eq. (9)], with all other parameters fixed at the values of the $\mathrm{C}_{60}$ fullerene. In this calculation, $R_{1}$ is estimated from the TDDFT value ${ }^{25}\left(534\right.$ bohrs $\left.^{3}\right)$ of the static dipole polarizability, while $R_{2}$ and $R_{3}$ are estimated from the classical relationship of Eq. (11), which yields $R_{1}=R_{2}$ $=R_{3}=R$ and thus $t_{1}=t_{2}=t_{3}=t$. Then, we take a variation of $t$ from 0 to $R_{l}$. For fixed $R$, the electron density decreases monotonically with increase of $t$, as shown by Eq. (36). From Fig. 2, we observe that the dynamic multipole polarizability rises fast when $t$ is much less than its value ( 2.8 bohrs) for $\mathrm{C}_{60}$, and then increases slowly with $t$, and finally reaches a maximum at $t=R$, where the density becomes lowest. The vdW coefficients for $t=R$ can be calculated from Eq. (37). For $l=1$ or $C_{6}$, this is just the well-known Slater-Kirkwood formula ${ }^{45}$

$$
C_{6, t_{1} \rightarrow R_{1}}=\frac{3}{2} \frac{\alpha_{1}^{\mathrm{A}}(0) \alpha_{1}^{\mathrm{B}}(0)}{\left(\alpha_{1}^{\mathrm{A}}(0) / N_{\mathrm{A}}\right)^{(1 / 2)}+\left(\alpha_{1}^{\mathrm{B}}(0) / N_{\mathrm{B}}\right)^{(1 / 2)}} .
$$

(ii) $N$ and $R_{l}$ are fixed, but $t_{l}$ varies down to 0 . Now we reverse the process by squeezing or compressing a shell of uniform density to a thin shell. Because $R$ is fixed at the values of $\mathrm{C}_{60}$, this process will make the density infinitely 

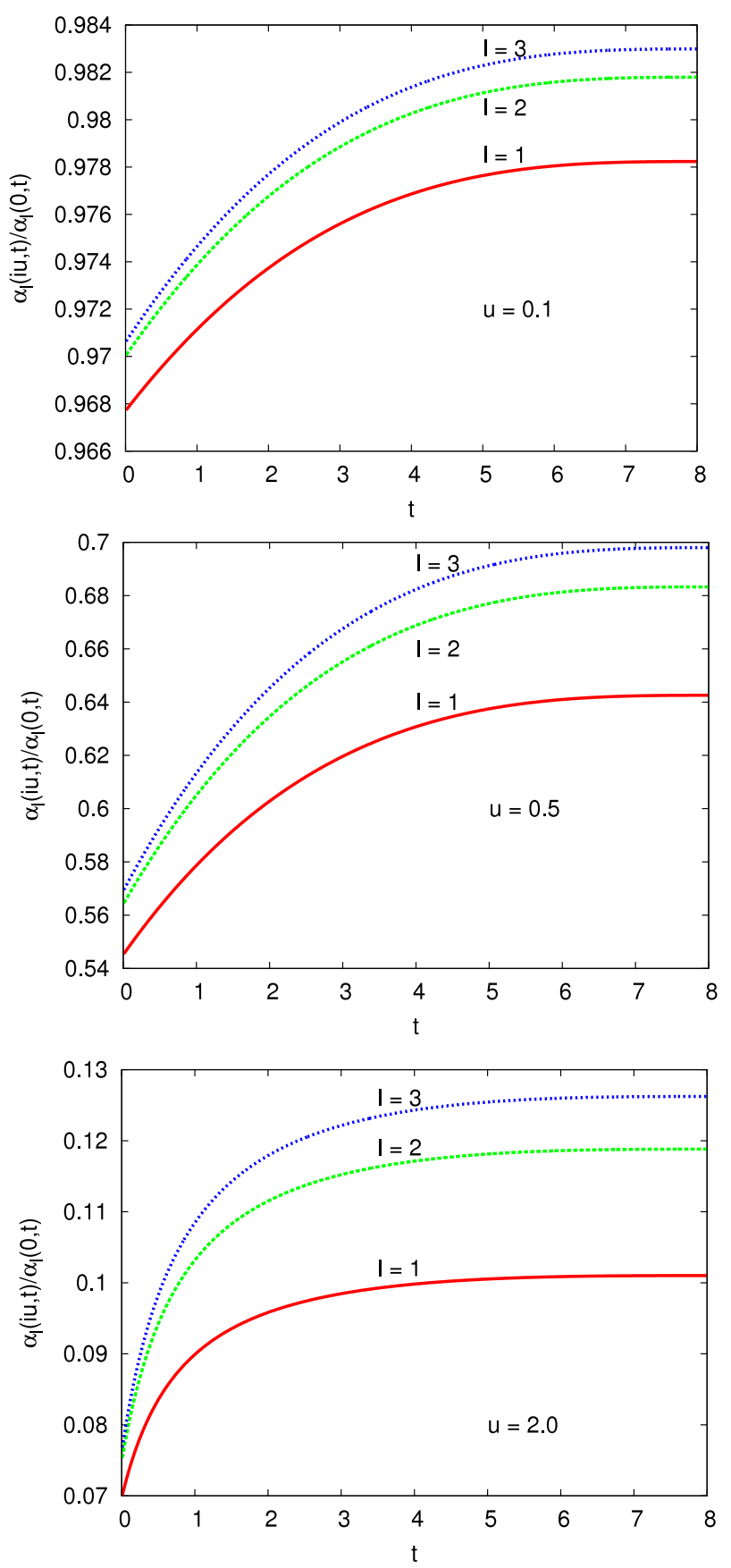

FIG. 2. Thickness dependence of the dynamic multipole polarizability with $l=1$ (dipole), $l=2$ (quadrupole), $l=3$ (octupole). All other parameters ( $N=240$ and $R_{l}=8.11$ bohrs) are at fixed values of $\mathrm{C}_{60}$.

high as $t_{l} \rightarrow 0$. In this limit, the dynamic multipole polarizability at high frequency is given by

$$
\alpha_{l}^{\mathrm{SFA}}(i u)=R_{l}^{2 l+1} \frac{\frac{N}{R_{l}^{3}} \frac{l(l+1)}{2 l+1}}{u^{2}+\frac{N}{R_{l}^{3}} \frac{l(l+1)}{2 l+1}} .
$$

Figure 3 shows $\alpha_{l}^{\mathrm{SFA}}\left(\mathrm{iu}\right.$ ) for $t=R, 2.8$ (value of $\mathrm{C}_{60}$ molecule), and 0. We observe from Fig. 3 that $\alpha_{1}^{\mathrm{SFA}}(i u)$ has a weak $t$-dependence for all orders $l$. This suggests that inflation or compression of systems by changing the shell thickness $t$ should not change the $\mathrm{vdW}$ coefficients noticeably.
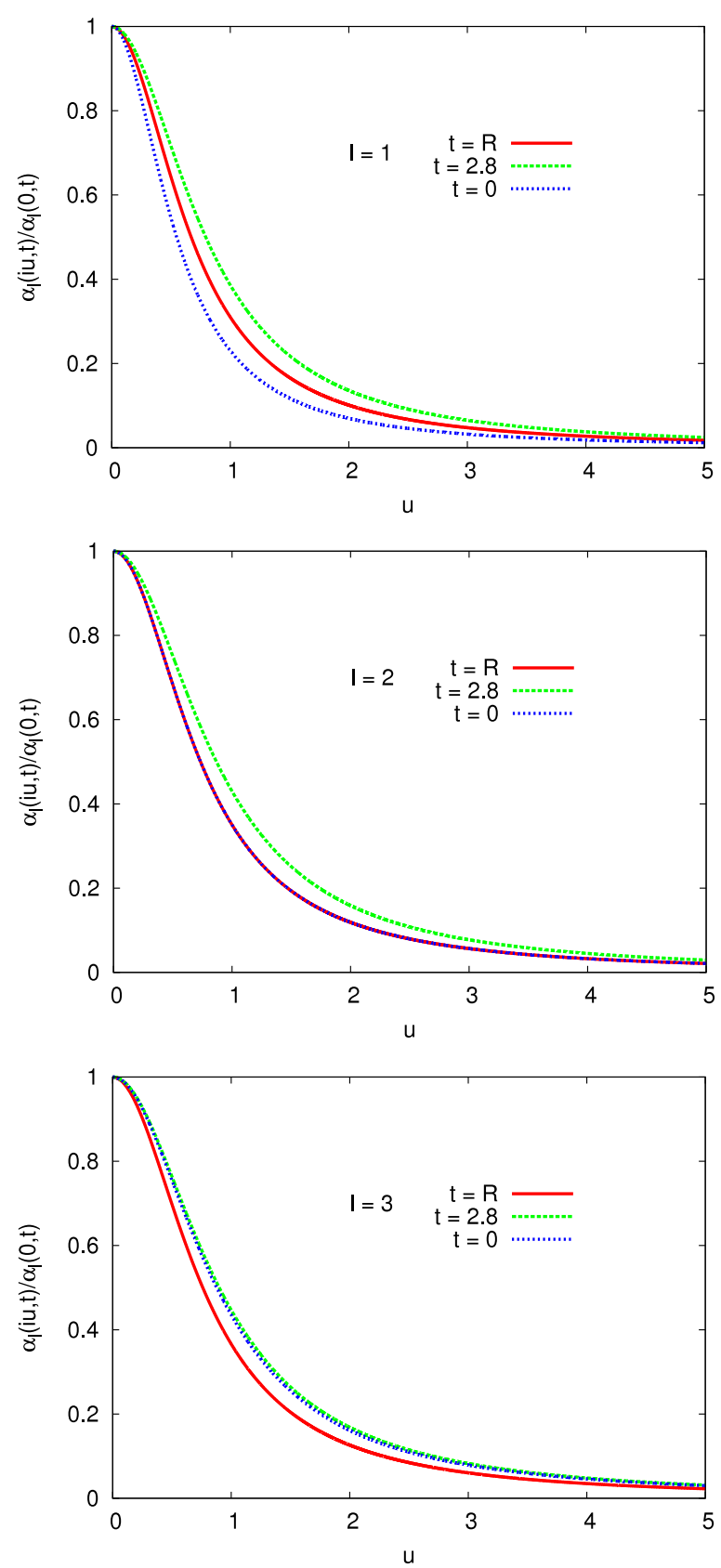

FIG. 3. Dynamic multipole polarizability as a function of $u$ for $t_{l}=R_{l}$ (solid sphere), $t=2.8$, and $t=0$ (thin shell). All other parameters $(N=240$ and $R_{l}=8.11$ bohrs) are at fixed values of $\mathrm{C}_{60}$.

The vdW coefficients for $t_{l} \rightarrow 0$ with $N$ and $R_{l}$ being held fixed can be calculated with the dynamic multipole polarizability of Eq. (41). They are given by

$$
\begin{aligned}
C_{2 k, t_{l} \rightarrow 0}^{\mathrm{AB}}= & \frac{(2 k-2) !}{4} \sum_{l_{1}=1}^{k-2} \frac{\alpha_{l_{1}}(0) \alpha_{l_{2}}(0)}{\left(2 l_{1}\right) !\left(2 l_{2}\right) !} /\left[\sqrt{\frac{\left(R_{l_{1}}^{\mathrm{A}}\right)^{3}}{N_{\mathrm{A}}} \frac{2 l_{1}+1}{l_{1}\left(l_{1}+1\right)}}\right. \\
& \left.+\sqrt{\frac{\left(R_{l_{2}}^{\mathrm{B}}\right)^{3}}{N_{\mathrm{B}}}} \frac{2 l_{2}+1}{l_{2}\left(l_{2}+1\right)}\right] .
\end{aligned}
$$

For the leading-order coefficient, we find

$$
C_{6, t_{1} \rightarrow 0}^{\mathrm{AB}}=\sqrt{\frac{2}{3}} C_{6, t_{1}=R_{1}}^{\mathrm{AB}} .
$$


This suggests that for a given system, squeezing the volume would reduce $C_{6}$ by a factor of $\sqrt{2 / 3}$. This is because when we squeeze a system to a small volume, the density becomes high and hard to deform.

(iii) $N$ and $n$ are fixed, but vary $t_{l}$ to 0 . Now we consider the case in which $N$ and the average density $n$ are held fixed. For $\mathrm{C}_{60}, N=240, R_{1}=R_{2}=R_{3}=R=8.1$, and $t_{1}$ $=t_{2}=t_{3}=t=2.8$. From Eq. (36), we have $V_{l}=1599$. When the shell collapses into a solid sphere (i.e, $t_{l}=R_{l}$ ), the vdW radius of the solid sphere drops from 8.1 to 7.3. On the other hand, when $t_{l} \rightarrow 0$, we have $R_{l}^{2}=V_{l} /\left(4 \pi t_{l}\right) \rightarrow$ $\infty$. For a given density and frequency, $\beta_{l}$ is a constant. The shape function takes the form of $\rho_{l}=1-(2 l+1) t_{l} / R_{l}$. Thus we have

$$
\alpha_{l}^{\mathrm{SFA}}(i u)=\frac{R_{l}^{2 l-2} \omega_{l}^{2}}{\omega_{l}^{2}+u^{2}} \frac{(2 l+1) R_{l}^{2} t_{l}}{1-\tilde{\beta}_{l}+\tilde{\beta}_{l}(2 l+1) R_{l}^{2} t_{l} / R_{l}^{3}},
$$

which is different from the one described by Eq. (41). This suggests that when electrons move further from the center, the polarizability will increase. In the static limit, $\tilde{\beta}_{l}=1$ and thus Eq. (44) correctly reduces to $\alpha_{l}^{\mathrm{SFA}}(i u)=R_{l}^{2 l+1}$. Unlike the static polarizability, the dynamic polarizability depends significantly upon $t_{l}$.

(iv) Increase the shell size to $R \rightarrow \infty$, while other parameters are fixed. Finally, we consider the case when the size of clusters approaches the extended electron gas. In this limit, the electron density becomes slowly varying within the nuclear framework. Thus, the two parameters in Eqs. (29) and (30) reduce to $d_{l} \approx 1$ and $R_{l} \approx\left[\alpha_{l}(0)\right]^{1 / 2 l+1}$. The usual pairwise-based models fail, due to the strong nonadditive nature of vdW interactions. Since the effective thickness $t_{l}$ is a constant (which is set to be 3.4 bohrs, independent of cluster $\operatorname{size}^{43}$ ), the shape function $\rho_{l}$ $\rightarrow 1-(2 l+1) t_{l} / R_{l}$. Thus, our model dynamic multipole polarizability becomes

$$
\alpha_{l}(i u)=\left(R_{l}^{2 l} \frac{\omega_{l}^{2}}{\omega_{l}^{2}+u^{2}}\right) \frac{(2 l+1) t}{1-\beta_{l}},
$$

where $R_{l}=\left[\alpha_{l}(0)\right]^{1 / 2 l+1}$. This situation is similar to case (iii) or Eq. (44).

\section{APPLICATION}

In this section, we apply the full hollow-sphere model and SFA to study the vdW coefficients between atoms and clusters and compare them to the classical shell model. From this study, we will see the role of the imposed correct conditions in vdW coefficients. Then, we apply our SFA to study the non-additivity of the vdW coefficients between metal clusters.

\section{A. Free atom pairs}

A major difference between SFA and the classical shell model is that the higher-order dynamic multipole polarizabilities in SFA satisfy the correct static limit, while those in the classical shell model do not. To see how this condition affects the performance of a model dynamic multipole polarizability, we apply SFA and the classical shell model to calculate the
vdW coefficients $C_{6}, C_{8}$, and $C_{10}$ for 78 atom pairs consisting of rare-gas atoms ( $\mathrm{He}, \mathrm{Ne}, \mathrm{Ar}, \mathrm{Kr}, \mathrm{Xe})$, hydrogen and alkali-metal atoms $(\mathrm{H}, \mathrm{Li}, \mathrm{Na}, \mathrm{K})$, and alkaline earth-metal atoms $(\mathrm{Be}, \mathrm{Mg}$, Ca). (For atoms, we set $t_{l}=R_{l}$ in both models.) The results are summarized in Table I. (See supplementary material ${ }^{42}$ for the detailed comparison for each atom pair.)

From Table I, we see that SFA is significantly better than the classical model, but less accurate than the full hollowsphere or solid-sphere model, which satisfies all important conditions. Table I also shows that the error in both SFA and CSM tends to increase from lower order to higher order, while that of the full hollow-sphere or solid-sphere model remains nearly the same. There are two reasons for this. First, in the full hollow-sphere model, the realistic local electron density is used, while in SFA and CSM, we model the electron density with the average valence electron density. Second, besides the recovery of the static limit, the full hollow-sphere model also respects the high-frequency limit. However, neither SFA nor CSM satisfies this exact condition. Nevertheless, SFA is much more accurate than CSM, because the former satisfies the correct static limit condition for each order, but the latter satisfies this condition only for dipole polarizability. As shown for cluster pairs below, the accuracy of CSM rapidly increases with cluster size and finally merges into SFA when the electron density becomes slowly varying, as cluster size approaches the bulk limit.

Table II shows dramatic errors of the classical relationship between static multipole polarizabilities of atoms. From Table II, we can observe that CSM tends to underestimate the higher-order static multipole polarizability for closed-shell rare-gas atoms, while the tendency reverses for open-shell atoms. From Tables S1-S3, we can observe that the error of CSM in the vdW coefficients is smaller for rare-gas dimers, though still too large, than for dimers or diatomics between open-shell atoms, such as alkali-metal atom pairs. A possible

TABLE II. Comparison of accurate static multipolar polarizabilities $\alpha_{l}(0)$ with CSM values for the quadrupole $(l=2)$ and octupole $(l=3)$ polarizabilities estimated from the classical relationship $\alpha_{l}^{\mathrm{csm}}(0)=\left[\alpha_{1}(0)\right]^{(2 l+1) / 3}$. Hartree atomic units are used.

\begin{tabular}{lccccc}
\hline \hline Atom & $\alpha_{1}(0)$ & $\alpha_{2}(0)$ & $\alpha_{3}(0)$ & $\alpha_{2}^{\text {csm }}(0)$ & $\alpha_{3}^{\text {csm }}(0)$ \\
\hline $\mathrm{H}$ & $4.50^{\mathrm{a}}$ & $15.0^{\mathrm{a}}$ & $131.25^{\mathrm{a}}$ & 12.27 & 33.43 \\
$\mathrm{He}$ & $1.38^{\mathrm{b}}$ & $2.331^{\mathrm{c}}$ & $9.932^{\mathrm{c}}$ & 1.711 & 2.120 \\
$\mathrm{Ne}$ & $2.67^{\mathrm{b}}$ & $7.33^{\mathrm{d}}$ & $42.1^{\mathrm{d}}$ & 5.139 & 9.890 \\
$\mathrm{Ar}$ & $11.1^{\mathrm{b}}$ & $51.84^{\mathrm{e}}$ & $534.85^{\mathrm{e}}$ & 55.23 & 274.8 \\
$\mathrm{Kr}$ & $16.8^{\mathrm{b}}$ & $98.43^{\mathrm{e}}$ & $1269.6^{\mathrm{e}}$ & 110.2 & 722.9 \\
$\mathrm{Xe}$ & $27.4^{\mathrm{b}}$ & $223.3^{\mathrm{d}}$ & $3640.6^{\mathrm{d}}$ & 249.0 & 2263 \\
$\mathrm{Li}$ & $164.1^{\mathrm{f}}$ & $1424^{\mathrm{g}}$ & $39688^{\mathrm{g}}$ & 4919 & 147430 \\
$\mathrm{Na}$ & $162.6^{\mathrm{f}}$ & $1878^{\mathrm{g}}$ & $55518^{\mathrm{g}}$ & 4844 & 144305 \\
$\mathrm{~K}$ & $290.2^{\mathrm{f}}$ & $5000^{\mathrm{g}}$ & $176940^{\mathrm{g}}$ & 12720 & 557563 \\
$\mathrm{Be}$ & $37.8^{\mathrm{b}}$ & $299.9^{\mathrm{c}}$ & $4765^{\mathrm{c}}$ & 425.7 & 4795 \\
$\mathrm{Mg}$ & $71.7^{\mathrm{b}}$ & $845.4^{\mathrm{c}}$ & $16772^{\mathrm{c}}$ & 1237 & 21357 \\
$\mathrm{Ca}$ & $158.6^{\mathrm{b}}$ & $3083^{\mathrm{d}}$ & $65170^{\mathrm{d}}$ & 4647 & 136157 \\
\hline \hline
\end{tabular}

${ }^{\mathrm{a}}$ From Ref. 46.

${ }^{\mathrm{b}}$ From Ref. 47

${ }^{\mathrm{c}}$ From Ref. 48

${ }^{\mathrm{d}}$ From Ref. 16

${ }^{\mathrm{e}}$ From Ref. 49

${ }^{\mathrm{f}}$ From Ref. 50.

${ }^{\mathrm{g}}$ From Ref. 51 
TABLE III. vdW coefficients $C_{6}\left(\times 10^{3}\right), C_{8}\left(\times 10^{5}\right)$, and $C_{10}\left(\times 10^{7}\right)$ (in Hartree atomic units) between alkali-metal atoms and $\mathrm{C}_{60}$. For atoms, we set $t_{l}=R_{l}$. The input static polarizabilities are taken from Ref. 29 for alkali-metal atoms and from Ref. 30 for $\mathrm{C}_{60}$. MARE = mean absolute relative error. $\mathrm{CSM}=$ classical shell model. Although reference values for $C_{8}$ and $C_{10}$ are unavailable, Table I suggests that SFA should be much more realistic than CSM for the higher-order coefficients. For the lowest-order coefficient $C_{6}, \mathrm{CSM}=\mathrm{SFA}$.

\begin{tabular}{|c|c|c|c|c|c|c|}
\hline & $C_{6}^{\mathrm{ref}}$ & $C_{6}^{\mathrm{SFA}}$ & $C_{8}^{\mathrm{SFA}}$ & $C_{10}^{\mathrm{SFA}}$ & $C_{8}^{\mathrm{CSM}}$ & $C_{10}^{\mathrm{CSM}}$ \\
\hline $\mathrm{Li}-\mathrm{C}_{60}$ & $8.07^{\mathrm{a}}$ & $9.14^{\mathrm{b}}$ & 18.32 & 29.88 & 22.78 & 46.64 \\
\hline $\mathrm{Na}-\mathrm{C}_{60}$ & $8.52^{\mathrm{a}}$ & 9.09 & 18.92 & 32.38 & 22.62 & 46.21 \\
\hline $\mathrm{K}-\mathrm{C}_{60}$ & $12.95^{\mathrm{a}}$ & 12.48 & 28.41 & 54.41 & 35.77 & 84.90 \\
\hline
\end{tabular}

MARE (\%)

7.9

a From Ref. 52.

${ }^{\mathrm{b}}$ From Ref. 30 .

reason is that for open-shell atoms, the electron density is more spread out into the classical forbidden or tail region than for closed-shell atoms, in which the electron density is more compact and thus more suitable for CSM.

\section{B. Atom-fullerene pairs}

We further apply SFA to calculate the vdW coefficients between $\mathrm{C}_{60}$ and alkali-metal atoms and compare them to the classical shell model. The results are shown in Table III. From Table III, we can see that the mean absolute relative deviation of the SFA values for $C_{6}$ from the TDDFT values ${ }^{52}$ is $8 \%$, smaller than that for atom-atom pairs. This is because the electron density in a fullerene is more slowly varying than that in an atom and thus can be modelled more accurately with SFA. However, there is a large discrepancy between SFA and CSM for $C_{8}$ and $C_{10}$. But this discrepancy is again smaller than that for atom pairs, due to the same reason.

\section{Non-additivity of vdW coefficients between metal clusters}

$N a_{\mathcal{N}}$ cluster. The sodium cluster is one of the most widely studied model systems. It can be well modeled by a jellium sphere. ${ }^{53}$ For the sodium and other metal clusters discussed below, we set $t_{l}=R_{l}$. Table IV shows comparison of the static multipole polarizability and vdW coefficients between SFA and the classical shell or solid-sphere model. From Table IV, we can see that there is a large discrepancy between SFA and CSM for the $\mathrm{Na}$ atom and the pair interaction $\mathrm{Na}-\mathrm{Na}$, but this discrepancy gets much smaller for the $\mathrm{Na}_{20}$ cluster and pair interaction $\mathrm{Na}_{20}-\mathrm{Na}_{20}$. For the $\mathrm{Na}_{92}$ cluster, SFA gives results that are nearly the same as those predicted by the classical shell model. The reason is that in small clusters, the electron density is not slowly varying so that the classical relationship [Eq. (12)] between the static multipole polarizabilities is not accurate. When cluster size increases, the electron density becomes more slowly varying. In this case, the classical relationship holds quite well. In this comparative study, the static multipole polarizabilities of $\mathrm{Na}_{20}$ and $\mathrm{Na}_{92}$ clusters are estimated based on the jellium model. Early calculations show that for $\mathrm{Na}_{20}$ or a larger cluster, to a good approximation, we may treat the sodium cluster as jellium.

$L i_{\mathcal{N}}, K_{\mathcal{N}}, N a_{\mathcal{N}}-K_{\mathcal{N}}$, and $A l_{\mathcal{N}}$ clusters. The intuitive atom pairwise interaction picture has been widely used to study the vdW interaction between two large systems. The popularity is based on the observation that this picture works quite well for small and middle-size molecules and accuracy for these systems can be tested with first-principles calculations. The simplest version of this picture is that each atom in a cluster of uniform chemical composition makes equal contribution to $C_{2 k}$, and that $C_{2 k}$ of any size pair interaction can be decomposed as a sum of all atom pairs formed by each atom in a cluster with each atom in another cluster. The vdW coefficients per atom pair for the pair interaction between two clusters can be found as $C_{2 k} / \mathcal{N}_{A} \mathcal{N}_{B}$, which is size-independent in a simple atom-pair interaction picture. However, it was recently found ${ }^{30}$ that the $\mathrm{vdW}$ coefficients per atom pair for clusters such as $\mathrm{Na}_{\mathcal{N}}, \mathrm{Ge}_{\mathcal{N}}$, and especially ${ }^{33}$ fullerenes $\mathrm{C}_{\mathcal{N}}$ exhibit a strong size-dependence. In particular, it was found that $C_{6}$ per atom pair displays a decreasing trend for $\mathrm{Na}_{\mathcal{N}}$, while the trend goes the opposite way for $\mathrm{Ge}_{\mathcal{N}}$ and $\mathrm{C}_{\mathcal{N}}$. This suggests that there exists strong non-additivity of longrange vdW interactions between nanostructures, leading to the failure of this picture for nanoclusters. It is interesting to note that $\mathrm{Ge}_{\mathcal{N}}$ has no usual electron delocalization as $\mathrm{Na}_{\mathcal{N}}$ and $\mathrm{C}_{\mathcal{N}}$ do, because the bulk limit of $\mathrm{Ge}_{\mathcal{N}}$ is a semiconductor.

Here, we apply SFA to study the evolution of the vdW coefficients per atom pair for $\mathrm{Li}_{\mathcal{N}}, \mathrm{K}_{\mathcal{N}}, \mathrm{Na}_{\mathcal{N}}-\mathrm{K}_{\mathcal{N}}$, and $\mathrm{Al}_{\mathcal{N}}$ clusters, whose input static polarizabilities per atom are listed in Table V. The results are tabulated in Tables VI-IX. In our calculations of $C_{6}$, accurate static dipole polarizabilities are used. From Tables VI and VII, we see that our model yields $C_{6}$

TABLE IV. Comparison of the static multipole polarizabilities $\alpha_{1}(0), \alpha_{2}(0)\left(\times 10^{3}\right)$, and $\alpha_{3}(0)\left(\times 10^{6}\right)$ for $\mathrm{Na}, \mathrm{Na}_{20}$, and $\mathrm{Na}_{92}$ clusters between the realistic values and the classical shell model $\left(t_{l}=R_{l}\right)$, and the vdW coefficients $C_{6}\left(\times 10^{4}\right), C_{8}\left(\times 10^{6}\right)$, and $C_{10}\left(\times 10^{8}\right)$ between two identical cluster pairs. Atomic units are used. The input static multipole polarizabilities for SFA are the accurate reference values. Taking SFA as the standard, note the dramatic improvement of CSM with increasing cluster size. For $C_{6}, \mathrm{CSM}=\mathrm{SFA}$.

\begin{tabular}{lcccccccccc}
\hline \hline Cluster & $\alpha_{1}(0)$ & $\alpha_{2}(0)$ & $\alpha_{2}^{\text {CSM }}(0)$ & $\alpha_{3}(0)$ & $\alpha_{3}^{\text {CSM }}(0)$ & $C_{6}^{\text {SFA }}$ & $C_{8}^{\text {SFA }}$ & $C_{8}^{\text {CSM }}$ & $C_{10}^{\text {SFA }}$ & $C_{10}^{\text {CSM }}$ \\
\hline $\mathrm{Na}$ & $162.6^{\mathrm{a}}$ & $1.878^{\mathrm{a}}$ & 4.844 & $0.0555^{\mathrm{a}}$ & 0.144 & 0.156 & 0.107 & 0.242 & 0.093 \\
$\mathrm{Na}_{20}$ & $1988^{\mathrm{b}}$ & $304.4^{\mathrm{b}}$ & 314.3 & $44.73^{\mathrm{a}}$ & 49.69 & 29.73 & $239.1^{\mathrm{c}}$ & $245.7^{\mathrm{c}}$ & 1570 & 1687 \\
$\mathrm{Na}_{92}$ & $7481^{\mathrm{b}}$ & $2845^{\mathrm{b}}$ & 2862 & $1068^{\mathrm{b}}$ & 1095 & 465.5 & 9262 & 9308 & 152300 & 154600 \\
\hline \hline
\end{tabular}

\footnotetext{
${ }^{\mathrm{a}}$ Taken from Ref. 29.

${ }^{\mathrm{b}}$ Evaluated from the jellium model with $\alpha_{l}=\left(R+\delta_{l}\right)^{2 l+1}$, where $R=N^{1 / 3} r_{s}$, with $r_{s}=4.0$, and $\delta$ value for each $l$ is estimated based on Ref. 53. For Na20, $\delta_{1}=1.716$, $\delta_{2}=1.636$, and $\delta_{3}=1.529$, while for Na92, $\delta_{1}=1.500, \delta_{2}=1.478$, and $\delta_{3}=1.433$.
}

${ }^{\mathrm{c}}$ Taken from Ref. 30. 
TABLE V. Input static dipole polarizability per atom (in atomic units) of $\mathrm{Li}_{n}, \mathrm{Na}_{n}, \mathrm{~K}_{n}$, and $\mathrm{Al}_{n}$ clusters.

\begin{tabular}{lccccccc}
\hline \hline Cluster & $\alpha_{1}(0)$ & Cluster & $\alpha_{1}(0)$ & Cluster & $\alpha_{1}(0)$ & Cluster & $\alpha_{1}(0)$ \\
\hline $\mathrm{Li}_{2}$ & $107.9^{\mathrm{a}}$ & $\mathrm{Na}_{2}$ & $129.8^{\mathrm{b}}$ & $\mathrm{K}_{2}$ & $241.6^{\mathrm{c}}$ & $\mathrm{Al}_{2}$ & $64.10^{\mathrm{d}}$ \\
$\mathrm{Li}_{4}$ & $85.60^{\mathrm{a}}$ & $\mathrm{Na}_{4}$ & $127.9^{\mathrm{b}}$ & $\mathrm{K}_{4}$ & $244.5^{\mathrm{c}}$ & $\mathrm{Al}_{4}$ & $49.68^{\mathrm{e}}$ \\
$\mathrm{Li}_{6}$ & $84.83^{\mathrm{a}}$ & $\mathrm{Na}_{6}$ & $124.0^{\mathrm{b}}$ & $\mathrm{K}_{6}$ & $218.0^{\mathrm{c}}$ & $\mathrm{Al}_{6}$ & $45.43^{\mathrm{e}}$ \\
$\mathrm{Li}_{8}$ & $70.26^{\mathrm{f}}$ & $\mathrm{Na}_{8}$ & $110.5^{\mathrm{b}}$ & $\mathrm{K}_{8}$ & $186.5^{\mathrm{c}}$ & $\mathrm{Al}_{8}$ & $44.00^{\mathrm{e}}$ \\
$\mathrm{Li}_{10}$ & $69.39^{\mathrm{f}}$ & $\mathrm{Na}_{10}$ & $105.3^{\mathrm{b}}$ & $\mathrm{K}_{10}$ & $200.7^{\mathrm{c}}$ & $\mathrm{Al}_{10}$ & $43.02^{\mathrm{e}}$ \\
$\mathrm{Li}_{12}$ & $68.78^{\mathrm{f}}$ & $\mathrm{Na}_{12}$ & $111.8^{\mathrm{b}}$ & $\mathrm{K}_{12}$ & $198.8^{\mathrm{c}}$ & $\mathrm{Al}_{12}$ & $40.25^{\mathrm{e}}$ \\
$\mathrm{Li}_{14}$ & $68.79^{\mathrm{f}}$ & $\mathrm{Na}_{14}$ & $137.7^{\mathrm{b}}$ & $\mathrm{K}_{14}$ & $198.7^{\mathrm{c}}$ & $\mathrm{Al}_{14}$ & $39.19^{\mathrm{e}}$ \\
$\mathrm{Li}_{16}$ & $65.19^{\mathrm{f}}$ & $\mathrm{Na}_{16}$ & & $\mathrm{~K}_{16}$ & $180.2^{\mathrm{c}}$ & $\mathrm{Al}_{16}$ & $38.98^{\mathrm{e}}$ \\
$\mathrm{Li}_{18}$ & $59.17^{\mathrm{f}}$ & $\mathrm{Na}_{18}$ & $95.83^{\mathrm{b}}$ & $\mathrm{K}_{18}$ & $169.4^{\mathrm{c}}$ & $\mathrm{Al}_{18}$ & $40.63^{\mathrm{e}}$ \\
$\mathrm{Li}_{20}$ & $61.00^{\mathrm{f}}$ & $\mathrm{Na}_{20}$ & $99.40^{\mathrm{b}}$ & $\mathrm{K}_{20}$ & $179.1^{\mathrm{c}}$ & $\mathrm{Al}_{20}$ & $38.09^{\mathrm{e}}$ \\
$\mathrm{Li}_{22}$ & $62.27^{\mathrm{f}}$ & & & & & $\mathrm{Al}_{22}$ & $37.70^{\mathrm{e}}$ \\
& & & & & $\mathrm{Al}_{24}$ & $38.61^{\mathrm{e}}$ \\
& & & & & $\mathrm{Al}_{26}$ & $39.65^{\mathrm{e}}$ \\
& & & & & $\mathrm{Al}_{28}$ & $39.04^{\mathrm{e}}$ \\
\hline \hline
\end{tabular}

\footnotetext{
${ }^{\mathrm{a}}$ From Ref. 54.

${ }^{\mathrm{b}}$ From Ref. 55 .

${ }^{\mathrm{c}}$ From Ref. 56.

${ }^{\mathrm{d}}$ From Ref. 58.

${ }^{\mathrm{e}}$ From Ref. 59.

${ }^{\mathrm{f}}$ From Ref. 61.
}

in good agreement with accurate reference values, in particular for larger cluster pairs, because the electron density in larger clusters is more slowly varying and thus can be modelled more accurately with SFA and CSM, compared to small cluster pairs such as $\mathrm{Li}_{2}$ (see Table VI), suggesting the consistently good accuracy of our model for clusters. From Tables VI-IX, we see that the vdW coefficients per atom pair for these metal clusters display a strong decreasing trend with cluster size, suggesting the strong non-additivity of vdW coefficients for metal clusters, as observed for sodium clusters. This confirms our early finding. ${ }^{30}$

As in Ref. 30, we find that $C_{6} / \mathcal{N}_{A} \mathcal{N}_{B}$ increases or decreases with $\mathcal{N}_{A}$ accordingly as $\alpha^{\mathrm{A}}(0) / \mathcal{N}_{A}$ does. A similar effect in passivated Si clusters was explained by Botti et al. ${ }^{60}$ in terms of a bond polarization model. A possible explanation

TABLE VI. vdW coefficients $C_{6}\left(\times 10^{3}\right)$ per atom pair (in atomic units) for Li cluster pairs $\left(t_{l}=R_{l}\right)$. The input of the accurate dipole polarizability for each cluster is taken from Table IV. The variation of $C_{6}$ per atom pair with cluster size in Tables Tables V-VIII is an indicator of strong non-additivity of the vdW interactions between nanoclusters. For $C_{6}, \mathrm{CSM}=\mathrm{SFA}$.

\begin{tabular}{lcc}
\hline \hline & $C_{6}^{\text {ref }} /$ atom pair & $C_{6}^{\mathrm{SF}} /$ atom pair \\
\hline $\mathrm{Li}_{2}-\mathrm{Li}_{2}$ & $0.684^{\mathrm{a}}$ & 0.8420 \\
$\mathrm{Li}_{4}-\mathrm{Li}_{4}$ & $0.512^{\mathrm{a}}$ & 0.594 \\
$\mathrm{Li}_{6}-\mathrm{Li}_{6}$ & $0.493^{\mathrm{a}}$ & 0.586 \\
$\mathrm{Li}_{8}-\mathrm{Li}_{8}$ & $0.405^{\mathrm{a}}$ & 0.442 \\
$\mathrm{Li}_{10}-\mathrm{Li}_{10}$ & $0.381^{\mathrm{a}}$ & 0.434 \\
$\mathrm{Li}_{12}-\mathrm{Li}_{12}$ & & 0.428 \\
$\mathrm{Li}_{14}-\mathrm{Li}_{14}$ & & 0.428 \\
$\mathrm{Li}_{16}-\mathrm{Li}_{16}$ & & 0.395 \\
$\mathrm{Li}_{18}-\mathrm{Li}_{18}$ & & 0.341 \\
$\mathrm{Li}_{20}-\mathrm{Li}_{20}$ & & 0.357 \\
$\mathrm{Li}_{22}-\mathrm{Li}_{22}$ & & 0.369 \\
\hline \hline
\end{tabular}

Taken from Ref. 54. for the decrease in static dipole polarizability per atom in the metals (Table V) is suggested by the stabilized jellium model: ${ }^{62}$ apart from small shell-structure oscillations, the polarizability is $\left(R_{+}+\delta\right)^{3}$, where $R_{+}$is the radius of the uniform positive background in the jellium or stabilized jellium models, and $\delta>0$ measures the spill-over of the electron density beyond the boundary $R_{+}$of the positive background, while the number of atoms is proportional to $R_{+}^{3}$, and the ratio $\left(R_{+}+\delta\right)^{3} / R_{+}^{3}$ decreases down to 1 with increasing $R_{+}$.

Our Tables VI-IX include the interactions between diatomic molecules (e.g., $\mathrm{Na}_{2}-\mathrm{Na}_{2}$ ). Because the densities of diatomic molecules can be far from spherical, ${ }^{63,64}$ our models are less reliable but still useful for the $\mathrm{vdW}$ interactions between diatomics (e.g., $\mathrm{Li}_{2}-\mathrm{Li}_{2}, \mathrm{~K}_{2}-\mathrm{K}_{2}$, and $\mathrm{Na}_{2}-\mathrm{K}_{2}$ ). The electron density in larger clusters can be more spherical and more slowly varying than in a diatomic molecule, and thus better suited to our models. But our hollow-sphere and SFA models work remarkably well (as shown earlier in Table I)

TABLE VII. vdW coefficients $C_{6}\left(\times 10^{3}\right)$ per atom pair (in atomic units) for $\mathrm{K}$ cluster pairs $\left(t_{l}=R_{l}\right)$. The input of the accurate dipole polarizability for each cluster is taken from Table IV. For $C_{6}, \mathrm{CSM}=\mathrm{SFA}$.

\begin{tabular}{lcc}
\hline \hline & $C_{6}^{\text {ref }} /$ atom pair & $C_{6}^{\text {SFA }} /$ atom pair \\
\hline $\mathrm{K}_{2}-\mathrm{K}_{2}$ & $3.000^{\mathrm{a}}$ & 2.816 \\
$\mathrm{~K}_{4}-\mathrm{K}_{4}$ & $2.923^{\mathrm{a}}$ & 2.866 \\
$\mathrm{~K}_{6}-\mathrm{K}_{6}$ & $2.553^{\mathrm{a}}$ & 2.414 \\
$\mathrm{~K}_{8}-\mathrm{K}_{8}$ & $2.317^{\mathrm{a}}$ & 1.910 \\
$\mathrm{~K}_{10}-\mathrm{K}_{10}$ & $2.08^{\mathrm{a}}$ & 2.132 \\
$\mathrm{~K}_{12}-\mathrm{K}_{12}$ & & 2.101 \\
$\mathrm{~K}_{14}-\mathrm{K}_{14}$ & & 2.101 \\
$\mathrm{~K}_{16}-\mathrm{K}_{16}$ & & 1.814 \\
$\mathrm{~K}_{18}-\mathrm{K}_{18}$ & & 1.653 \\
$\mathrm{~K}_{20}-\mathrm{K}_{20}$ & & 1.798 \\
\hline \hline
\end{tabular}

${ }^{\mathrm{a}}$ Taken from Ref. 54. 
TABLE VIII. vdW coefficients $C_{6}\left(\times 10^{3}\right)$ per atom pair (in atomic units) between $\mathrm{Na}$ and $\mathrm{K}$ clusters $\left(t_{l}=R_{l}\right)$. The input of the accurate dipole polarizability for each cluster is taken from Table IV. For $C_{6}, \mathrm{CSM}=\mathrm{SFA}$.

\begin{tabular}{lcc}
\hline \hline & $C_{6}^{\text {ref }} /$ atom pair & $C_{6}^{\text {SFA }} /$ atom pair \\
\hline $\mathrm{Na}_{2}-\mathrm{K}_{2}$ & $1.750^{\mathrm{a}}$ & 1.749 \\
$\mathrm{Na}_{4}-\mathrm{K}_{4}$ & $1.790^{\mathrm{a}}$ & 1.744 \\
$\mathrm{Na}_{6}-\mathrm{K}_{6}$ & $1.540^{\mathrm{a}}$ & 1.568 \\
$\mathrm{Na}_{8}-\mathrm{K}_{8}$ & $1.251^{\mathrm{a}}$ & 1.280 \\
$\mathrm{Na}_{10}-\mathrm{K}_{10}$ & $1.344^{\mathrm{a}}$ & 1.300 \\
$\mathrm{Na}_{12}-\mathrm{K}_{12}$ & $1.330^{\mathrm{a}}$ & 1.353 \\
$\mathrm{Na}_{14}-\mathrm{K}_{14}$ & $1.292^{\mathrm{a}}$ & 1.411 \\
$\mathrm{Na}_{18}-\mathrm{K}_{18}$ & $1.074^{\mathrm{a}}$ & 1.069 \\
$\mathrm{Na}_{20}-\mathrm{K}_{20}$ & $1.202^{\mathrm{a}}$ & 1.145 \\
\hline \hline
\end{tabular}

aTaken from Ref. 57.

even for free atoms, which are perfectly spherical but furthest from the slowly varying limit.

\section{RELATIVE IMPORTANCE OF $\boldsymbol{C}_{\mathbf{8}}$ AND $\boldsymbol{c}_{10}$}

According to MBPT, the vdW energy is a sum of an infinite power series of the inverse $d$ given by Eq. (1). If $d$ is large enough as in a core-core pair interaction for alkali metals, the asymptotic expansion of Eq. (1) is valid and directly applicable. We have found ${ }^{2}$ that at the equilibrium distance, the higher-order contribution can be as large as $50 \%$ of the leadingorder term for alkali metals. We have found here (Fig. 4) that for a $\mathrm{C}_{60}$ molecular crystal at the equilibrium distance, $C_{6} / d^{6}: C_{8} / d^{8}: C_{10} / d^{10}=1.0: 0.9: 0.7$. This ratio may vary from one molecular solid to another. Here is just an example showing the relative importance of higher-order contributions in the vdW energy. To get a better understanding, we calculate the contribution of each term in Eq. (1) for two molecular crystals $\mathrm{C}_{60}$ and $\mathrm{C}_{70}$. The variation of each term with the separation distance is plotted in Fig. 4. From Fig. 4, we observe that at the equilibrium distance, the contribution of the $C_{8}$ term

TABLE IX. vdW coefficients $C_{6}\left(\times 10^{3}\right)$ per atom pair (in atomic units) for Al cluster pairs $\left(t_{l}=R_{l}\right)$. The input of the accurate dipole polarizability for each cluster is taken from Table IV. For $C_{6}, \mathrm{CSM}=\mathrm{SFA}$.

\begin{tabular}{lc}
\hline \hline & $C_{6}^{\text {SFA }} /$ atom pair \\
\hline $\mathrm{Al}_{2}-\mathrm{Al}_{2}$ & 0.667 \\
$\mathrm{Al}_{4}-\mathrm{Al}_{4}$ & 0.455 \\
$\mathrm{Al}_{6}-\mathrm{Al}_{6}$ & 0.398 \\
$\mathrm{Al}_{8}-\mathrm{Al}_{8}$ & 0.379 \\
$\mathrm{Al}_{10}-\mathrm{Al}_{10}$ & 0.367 \\
$\mathrm{Al}_{12}-\mathrm{Al}_{12}$ & 0.332 \\
$\mathrm{Al}_{14}-\mathrm{Al}_{14}$ & 0.319 \\
$\mathrm{Al}_{16}-\mathrm{Al}_{16}$ & 0.316 \\
$\mathrm{Al}_{18}-\mathrm{Al}_{18}$ & 0.336 \\
$\mathrm{Al}_{20}-\mathrm{Al}_{20}$ & 0.305 \\
$\mathrm{Al}_{22}-\mathrm{Al}_{22}$ & 0.301 \\
$\mathrm{Al}_{24}-\mathrm{Al}_{24}$ & 0.312 \\
$\mathrm{Al}_{26}-\mathrm{Al}_{26}$ & 0.324 \\
$\mathrm{Al}_{28}-\mathrm{Al}_{28}$ & 0.317 \\
$\mathrm{Al}_{30}-\mathrm{Al}_{30}$ & 0.300 \\
\hline \hline
\end{tabular}
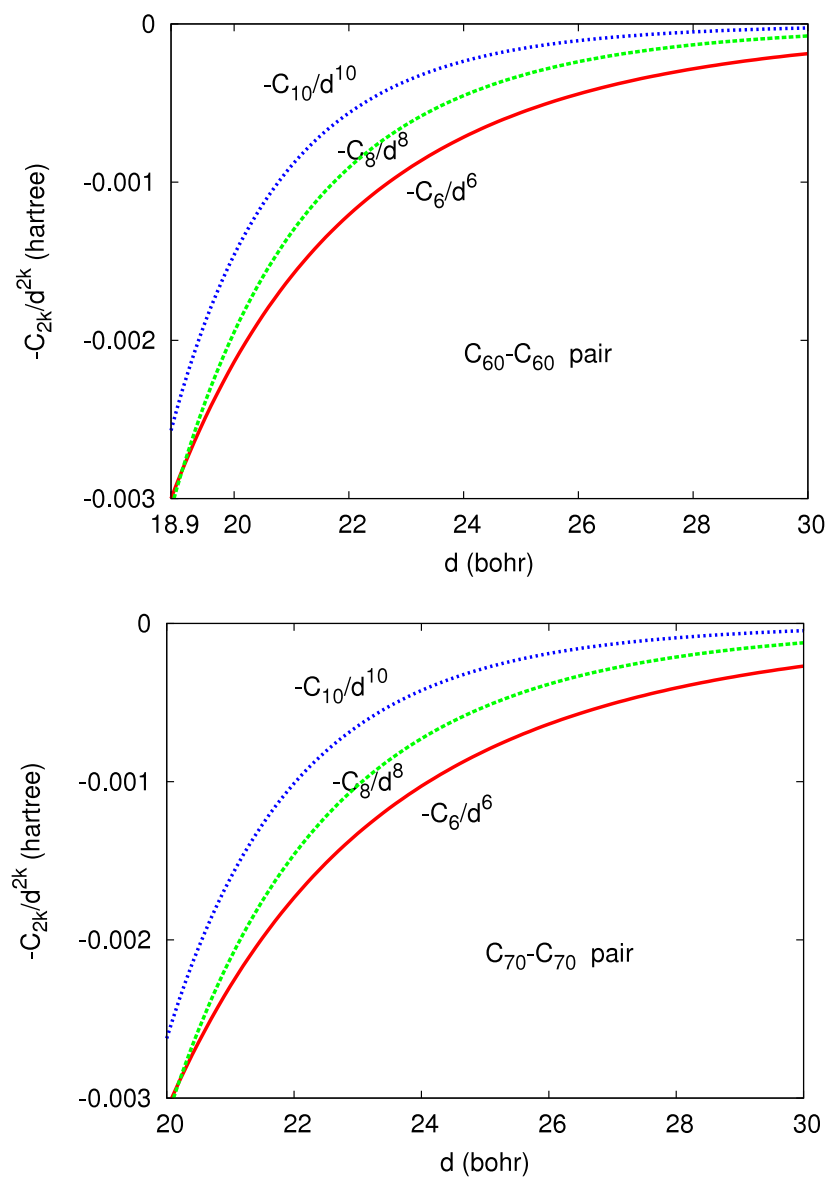

FIG. 4. Contributions of the leading and high-order terms to the vdW energy $C_{2 k}$ as a function of separation distance between the centers of two density fragments. Upper panel for $\mathrm{C}_{60}-\mathrm{C}_{60}$ pair, and lower panel for $\mathrm{C}_{70}-\mathrm{C}_{70}$ pair. The equilibrium distance is 18.9 bohrs for $\mathrm{C}_{60}$ solid (upper panel) and 20.0 bohrs for $\mathrm{C}_{70}$ solid (lower panel). $\left(C_{6}, C_{8}\right.$, and $C_{10}$ are SFA values from Ref. 30.).

is nearly the same as that of the $C_{6}$ term, while the $C_{10}$ term is slightly less important. When the separation $d$ increases, all the contributions decay fast, but the $C_{10}$ term decays fastest, followed by $C_{8}$ and then by $C_{6}$.

\section{CONCLUSION}

In summary, we have studied the properties of the dynamic multipole polarizability with the hollow-sphere model and applied it to study the vdW coefficients for systems that allow for a cavity. Compared to the classical shell model, which requires only the static dipole polarizability as input, the hollow-sphere model needs the static multipole polarizability as input, but it provides a more accurate description in particular for systems smaller than nanoscale. While there is little difference between these two models when applied to large systems, a noticeable difference has been observed for $C_{10}$ for interactions with fullerenes. We have further shown that higher-order contributions can be more important than the leading-order term in some cases such as fullerenes in the solid phase. For an accurate simulation of the vdW effect, considering $C_{6}$ alone may not be enough. The large size and the strong nonadditivity $33,65,66,30$ of $\mathrm{vdW}$ interactions present 
a major challenge to physicists and chemists in the modeling of nanostructures, while the present approach may reduce this difficulty.

\section{ACKNOWLEDGMENTS}

We thank Dr. Bing Xiao for helpful communications. This material was based on the work supported by NSF grants: Grant Nos. DMR-1305135 (JPP) and CHE 1261918 (JT). JT acknowledges support from the Air Force Office of Scientific Research, Air Force Materiel Command, USAF, under Grant No. FA9550-10-1-0248 (via Andrew M. Rappe). Computational support was provided by the HPCMO and the NERSC. GES is a Welch Foundation Chair (C-0036).

${ }^{1}$ I. G. Kaplan, Intermolecular Interactions: Physical Picture, Computational Methods and Model Potentials (John Wiley and Sons Ltd., England, 2006).

${ }^{2}$ J. Tao, J. P. Perdew, and A. Ruzsinszky, Phys. Rev. B 81, 233102 (2010).

${ }^{3}$ G.-X. Zhang, A. Tkatchenko, J. Paier, H. Appel, and M. Scheffler, Phys. Rev. Lett. 107, 245501 (2011).

${ }^{4}$ J. J. Rehr, E. Zaremba, and W. Kohn, Phys. Rev. B 12, 2062 (1975).

${ }^{5}$ A. Bondi, J. Chem. Eng. Data 8, 371 (1963).

${ }^{6}$ B. Santra, J. Klimes, D. Alfe, A. Tkatchenko, B. Slater, A. Michaelides, R. Car, and M. Scheffler, Phys. Rev. Lett. 107, 185701 (2011).

${ }^{7}$ R. A. DiStasio, O. A. von Lilienfeld, and A. Tkatchenko, Proc. Natl. Acad. Sci. U. S. A. 109, 14791 (2012).

${ }^{8}$ Physical Adsorption: Experiment, Theory and Application, edited by J. Fraissard and C. W. Conner (Kluwer Academic Publishers, Dordrecht, 1997).

${ }^{9}$ G. Vidali, G. Ihm, H. Y. Kim, and M. W. Cole, Surf. Sci. Rep. 12, 133 (1991).

${ }^{10}$ J. Tao and A. M. Rappe, Phys. Rev. Lett. 112, 106101 (2014).

${ }^{11}$ A. W. C. van den Berg and C. O. Areán, Chem. Commun. 2008(668).

${ }^{12}$ A. Dalgarno and G. A. Victor, Proc. Phys. Soc. 90, 605 (1967).

${ }^{13}$ S. H. Patil and K. T. Tang, J. Chem. Phys. 106, 2298 (1997).

${ }^{14}$ D. Spelsberg, T. Lorenz, and W. Meyer, J. Chem. Phys. 99, 7845 (1993).

${ }^{15}$ K. T. Tang, J. M. Norbeck, and P. R. Certain, J. Chem. Phys. 64, 3063 (1976).

${ }^{16}$ A. J. Thakkar, H. Hettema, and P. E. S. Wormer, J. Chem. Phys. 97, 3252 (1992).

${ }^{17}$ J. E. Rice, P. R. Taylor, T. J. Lee, and J. Almlöf, J. Chem. Phys. 94, 4972 (1991).

${ }^{18}$ L. Bytautas and K. Ruedenberg, J. Chem. Phys. 130, 204101 (2009).

${ }^{19}$ V. A. Dzuba, V. V. Flambaum, and M. G. Kozlov, Phys. Rev. A 54, 3948 (1996).

${ }^{20}$ S. G. Porsev and A. Derevianko, Phys. Rev. A 65, 020701(R) (2002).

${ }^{21}$ E. Zaremba and W. Kohn, Phys. Rev. B 13, 2270 (1976).

${ }^{22}$ V. P. Osinga, S. J. A. van Gisbergen, J. G. Snijders, and E. J. Baerends, J. Chem. Phys. 106, 5091 (2007).

${ }^{23}$ X. Chu and A. Dalgarno, J. Chem. Phys. 121, 4083 (2004).

${ }^{24}$ S. J. A. van Gisbergen, J. G. Snijders, and E. J. Baerends, J. Chem. Phys. 103, 9347 (1995).

${ }^{25}$ M. van Faassen, L. Jensen, J. A. Berger, and P. L. de Boeij, Chem. Phys. Lett. 395, 274 (2004).

${ }^{26}$ A. D. Becke and E. R. Johnson, J. Chem. Phys. 127, 154108 (2007).

${ }^{27}$ N. A. Lima, J. Chem. Phys. 132, 014110 (2010).

${ }^{28}$ O. A. Vydrov and T. Van Voorhis, Phys. Rev. A 81, 062708 (2010).

${ }^{29}$ J. Tao, J. P. Perdew, and A. Ruzsinszky, Proc. Natl. Acad. Sci. U. S. A. 109, 18 (2012)

${ }^{30}$ J. Tao and J. P. Perdew, J. Chem. Phys. 141, 141101 (2014).
${ }^{31}$ J. Tao, J. P. Perdew, and A. Ruzsinszky, Int. J. Mod. Phys. B 27, 1330011 (2013).

32 J. P. Perdew, J. Tao, P. Hao, A. Ruzsinszky, G. I. Csonka, and J. M. Pitarke, J. Phys.: Condens. Matter 24, 424207 (2012).

${ }^{33}$ A. Ruzsinszky, J. P. Perdew, J. Tao, G. I. Csonka, and J. M. Pitarke, Phys. Rev. Lett. 109, 233203 (2012).

${ }^{34}$ J. P. Perdew, A. Ruzsinszky, J. Sun, S. Glindmeyer, and G. I. Csonka, Phys. Rev. A 86, 062714 (2012).

${ }^{35}$ Y. Zhao and D. G. Truhlar, J. Chem. Phys. 125, 194101 (2006).

${ }^{36}$ J. Sun, B. Xiao, Y. Feng, R. Haunschild, P. Hao, A. Ruzsinszky, G. I. Csonka, G. E. Scuseria, and J. P. Perdew, Phys. Rev. Lett. 111, 106401 (2013).

${ }^{37}$ M. Dion, H. Rydberg, E. Schröder, D. C. Langreth, and B. I. Lundqvist, Phys. Rev. Lett. 92, 246401 (2004).

${ }^{38}$ S. Grimme, J. Antony, S. Ehrlich, and H. Krieg, J. Chem. Phys. 132, 154104 (2010).

${ }^{39}$ A. Tkatchenko, A. Ambrosetti, and R. A. DiStasio, J. Chem. Phys. 138, 074106 (2013).

${ }^{40}$ A. Tkatchenko and M. Scheffler, Phys. Rev. Lett. 102, 073005 (2009).

${ }^{41}$ A. A. Lucas, L. Henrard, and P. Lambin, Phys. Rev. B 49, 2888 (1994).

${ }^{42}$ See supplementary material at http://dx.doi.org/10.1063/1.4905259 for detailed comparisons between SFA and CSM for each atom pair..

${ }^{43}$ G. K. Gueorguiev, J. M. Pacheco, and D. Tománek, Phys. Rev. Lett. 92, 215501 (2004)

${ }^{44}$ A. J. Stone, The Theory of Intermolecular Forces, 2nd ed. (Clarendon Press, Oxford, 2013).

${ }^{45}$ CRC Handbook of Chemistry and Physics, 76th ed., edited by D. R. Lide (CRC, Boca Raton, 1995).

${ }^{46}$ A. Z. Tang and F. T. Chan, Phys. Rev. A 33, 3671 (1986).

${ }^{47} \mathrm{P}$. Schwerdtfeger, "Atomic static dipole polarizabilities," in Atoms, Molecules and Clusters in Electric Fields. Theoretical Approaches to the Calculation of Electric Polarizability, edited by G. Maroulis (World Scientific, London, 2006), Vol. 1, pp. 1-32.

${ }^{48}$ G. Figari, G. F. Musso, and V. Magnasco, Mol. Phys. 50, 1173 (1983).

${ }^{49}$ D. Spelsberg and W. Meyer, J. Phys. Chem. 100, 14637 (1996).

${ }^{50}$ A. Derevianko, W. R. Johnson, M. S. Safronova, and I. F. Babb, Phys. Rev. Lett. 82, 3589 (1999).

${ }^{51}$ M. Marinescu, H. R. Sadeghpour, and A. Dalgarno, Phys. Rev. A 49, 982 (1994).

${ }^{52}$ A. Banerjee, J. Autschbach, and A. Chakrabarti, Phys. Rev. A 78, 032704 (2008).

${ }^{53}$ L. Serra, F. Garcias, M. Barranco, J. Navarro, L. C. Balbás, A. Rubio, and A. Mañanes, J. Phys.: Condens. Matter 1, 10391 (1989).

${ }^{54}$ A. Jiemchooroj, B. E. Sernelius, and P. Norman, J. Comput. Methods Sci. Eng. 7, 475 (2007); available at http://liu.diva-portal.org/smash/record.jsf? pid=diva2\%3A16912\&dswid $=-3519$.

${ }^{55}$ A. Jiemchooroj, P. Norman, and B. E. Sernelius, J. Chem. Phys. 125, 124306 (2006).

${ }^{56}$ A. Banerjee, T. K. Ghanty, and A. Chakrabarti, J. Phys. Chem. A 112, 12303 (2008).

${ }^{57}$ A. Banerjee, A. Chakrabarti, and T. Ghanty, Int. J. Quantum Chem. 109, 1376 (2009).

${ }^{58}$ P. Milani, I. Moullet, and W. A. de Heer, Phys. Rev. A 42, 5150 (1990).

${ }^{59}$ M. Alipour and A. Mohajeri, J. Phys. Chem. A 114, 12709 (2010).

${ }^{60}$ S. Botti, A. Castro, X. Andrade, A. Rubio, and M. A. L. Marques, Phys. Rev. B 78, 035333 (2008).

${ }^{61}$ R. R. Zope, T. Baruah, and M. R. Pederson, J. Comput. Methods Sci. Eng. 2, 1 (2007); available at http://arxiv.org/pdf/0706.0555.pdf.

${ }^{62}$ M. Seidl and J. P. Perdew, Phys. Rev. B 50, 5744 (1994).

${ }^{63}$ P. E. S. Wormer, H. Hettema, and A. J. Thakkar, J. Chem. Phys. 98, 7140 (1993).

${ }^{64}$ M. Bartomomei, E. Carmona-Novillo, M. I. Herńandez, J. CamposMartínez, and R. Herńandez-Lamoneda, J. Comput. Chem. 32, 279 (2010).

${ }^{65}$ J. Kauczor, P. Norman, and W. A. Saidi, J. Chem. Phys. 138, 114107 (2013).

${ }^{66}$ V. V. Gobre and A. Tkatchenko, Nat. Commun. 4, 2341 (2013). 